lution pulmonary artery (STPA) catheter in reducing risk of systemic infections associated with pulmonary artery catheterization. The shielded catheter is covered completely during balloon testing, preparation, and insertion.

To assess the value of this STPA catheter in the prevention of systemic infections associated with pulmonary artery catheterization, they conducted a randomized, prospective study over an 18-month period. The patients were randomly assigned to two groups, of which one received a standard pulmonary artery catheter and the other, the STPA catheter. The diagnosis of systemic infection was based on recovery of the same organism from the thermodilution catheter (TC) and from blood samples; absence of any other infectious focus; and improvement or resolution of clinical evidence of infection after removal of the TC. A total of 166 TCs were randomized in 150 patients.

Eight cases of systemic infection were diagnosed in the standard TC group, versus none in the STPA catheter group $(P<.002)$. No cases of systemic infections occurred in those patients who had their TC for less than 4 days. A shielded pulmonary artery catheter may reduce the risk of systemic infections associated with prolonged pulmonary artery catheterization.

FROM: Cohen Y, Fosse JP, Karoubi P, Reboul-Marty J, Dreyfuss D, Hoang P, et al. The "hands-off" catheter and the prevention of systemic infections associated with pulmonary artery catheter: a prospective study. Am J Respir Crit Care Med 1998;157(1):284-287.

\section{Infectious Complications Associated with Histamine 2-Receptor Antagonists}

Investigators from the Department of Surgery, University of Washington, and Harborview Medical Center in Seattle conducted a study to determine the impact of histamine $_{2}\left(\mathrm{H}_{2}\right)$-receptor antagonist use on the occurrence of infectious complications in severely injured patients. Some previous studies suggest an increased risk of nosocomial pneumonia associated with the use of $\mathrm{H}_{2}-$ receptor blockade in critically ill patients, but other investigations suggest an immune-enhancing effect of $\mathrm{H}_{2}$ receptor antagonists. The purpose of this study was to determine whether $\mathrm{H}_{2}$-receptor antagonist use affects the overall incidence of infectious complications.

Patients enrolled in a randomized trial comparing ranitidine with sucralfate for gastritis prophylaxis were examined for all infectious complications during their hospitalization. Data on the occurrence of pneumonia were collected prospectively, and other infectious complications were obtained retrospectively from the medical record.

The relative risk of infectious complications associated with ranitidine use and total infectious complications were analyzed. Of patients whose 96 charts were available for review, sucralfate was given to 47 , and 49 received ranitidine. Ranitidine use was associated with a 1.5-fold increased risk of developing any infectious complication (37/47 vs 26/47). Infectious complications totaled 128 in the ranitidine-treated group and 50 in the sucralfate-treated group $(P=.0014)$. These differences remained after excluding catheter-related infections $(P=.0042)$ and secondary bacteremia $(P=.0046)$. The authors concluded that ranitidine use in severely injured patients is associated with a statistically significant increase in overall infectious complications when compared with sucralfate. These results indicate that ranitidine should be avoided where possible in the prophylaxis of stress gastritis.

FROM: O'Keefe GE, Gentilello LM, Maier RV. Incidence of infectious complications associated with the use of histamine 2 -receptor antagonists in critically ill trauma patients. Ann Surg 1998;227:120-125.

\section{Dialysis-Associated Diseases in the United States}

The CDC's Hospital Infections Program and Hepatitis Branch have been conducting surveillance of dialysisassociated diseases in hemodialysis centers since the 1980s. Results of a 1995 survey on hemodialysis-associated disease and infection control practices in chronic hemodialysis centers in the United States were published recently.

A total of 2,647 centers responded, representing 224,954 patients and 54,194 staff members. Seventy-seven percent of centers reported that they reused disposable dialyzers. At the end of $1995,65 \%$ of patients were treated with an arteriovenous graft, $22 \%$ with an arteriovenous fistula, and $13 \%$ with a temporary or permanent central catheter. By the end of 1995, at least three doses of hepatitis $B$ vaccine had been administered to $35 \%$ of patients and to $82 \%$ of staff members.

Acute infection with the hepatitis B virus (HBV) occurred in $0.06 \%$ of patients and was more likely to be reported by centers with lower proportions of patients vaccinated against HBV. The prevalence of antibody to hepatitis $\mathrm{C}$ virus was $10.4 \%$ among patients and $2.0 \%$ among staff.

At least one patient with vancomycin-resistant enterococci was reported by $11.5 \%$ of centers, more commonly by hospitals (vs freestanding centers not located in hospitals) and government centers, and centers located in certain geographic areas. Vancomycin was received by $7.2 \%$ of patients in December 1995. The percentage of centers reporting patients with other pathogens was $7.9 \%$ for active TB, 39\% for HIV, and 40\% for methicillin-resistant Staphylococcus aureus.

FROM: Tokars JI, Miller ER, Alter MJ, Arduino MJ. National surveillance of dialysis associated diseases in the United States, 1995. American Society for Artificial Internal Organs Journal 1998;44:98-107.

\section{Surveillance of Unexplained Illness and Death}

Researchers from the CDC's Emerging Infections Program (EIP) recently summarized the findings from population-based surveillance studies being conducted by 